\title{
Improvements in Interface Design through Implicit Modeling
}

\author{
Patrick K.A. Wollner, Ian Hosking, Patrick M. Langdon, and P. John Clarkson \\ Engineering Design Centre, Department of Engineering, University of Cambridge \\ \{pkaw2, imh29,pm124,pjc10\}@cam.ac.uk
}

\begin{abstract}
Touchscreen devices are often limited by the complexity of their user interface design. In the past, iterative design processes using representative user groups to test prototypes were the standard method for increasing the inclusivity of a given design, but cognitive modeling has potential to be an alternative to rigorous user testing. However, these modeling approaches currently have many limitations, some of which are based on the assumptions made in translating a User Interface (UI) into a definition file that cognitive modeling frameworks can process. This paper discusses these issues and postulates potential approaches to improvements to the translation procedure.
\end{abstract}

Keywords: inclusive design, universal design, cognitive modeling, cognitive architectures.

\section{Introduction}

While accessibility guidelines exist that reduce the barriers to accessing Information and Communications Technology (ICT), guidelines alone do not guarantee a more inclusive 11 design. In the past, iterative design processes using representative user groups to test prototypes were the standard method for increasing the inclusivity of a given design, but cognitive modeling has recently become a feasible alternative to rigorous user testing. However, these modeling approaches currently have many limitations, some of which are based on the assumptions made in translating a UI into a definition file that cognitive modeling frameworks can process. Cognitive modeling tools require information about the interface to be provided in a certain format (hereafter referred to as sampling), but this type of format simplifies the interface in such a way that some information about it is lost (this process is referred to as implicit modeling).

An iterative inclusive design approach can only be realised if incremental versions of the interface are tested with actual representative users or, as has recently become possible, through modeling frameworks that employ cognitive

\footnotetext{
${ }^{1}$ Inclusivity in this context is defined by the BSI 2]: "The design of mainstream products and/or services that are accessible to, and usable by, as many people as reasonably possible [...] without the need for special adaptation or specialised design."
}

C. Stephanidis and M. Antona (Eds.): UAHCI/HCII 2013, Part I, LNCS 8009, pp. 127-136, 2013.

(C) Springer-Verlag Berlin Heidelberg 2013 
modeling architectures such as ACT-R [1] or CASCaS [16 to represent human cognitive processes. These frameworks require the translation of interface features into a definition file that segments both the visual and conceptual elements of the interface into a form that is machine-interpretable by cognitive architectures.

Using a modeling approach for user testing provides multiple benefits, such as allowing the designer to (i) complete quicker iterations as a partial substitute for or in addition to time-consuming participant recruitment and testing, and (ii) utilise a tool that "learns" which prior knowledge the virtual user may have, a key element in the ability to interact with a new device/interface [14]. However, modeling approaches are currently limited by the loss of information through sampling: the process of translating an interface into a generic descriptive format. A cognitive framework can interpret this translation [21, but the drawback is that it results in a loss of detailed information about the interface.

While (i) and (ii) above provide obvious reasons (e.g. time, cost and convenience) for more widespread use of modeling tools by interface designers, the limitations currently still outweigh the benefits. This is characterised by the limited uptake of software packages that employ this methodology in industry applications. Furthermore, in the short term, no significant improvements in the accuracy of cognitive modeling frameworks are expected [19]. Hence, an increase in the accuracy of "sampling" an interface into a definition file is a feasible and often overlooked method of improving cognitive modeling approaches to interface design.

Sampling the interface may also be described as an "implicit" level of modeling. Currently, it is integrated into modeling tools through KLM-GOMS [13, a keystroke-level model which segments the interface into cognitively-processable actions, which in turn can be interpreted by cognitive architectures. Advancements can be achieved by critically assessing the limitations of the KLM approach and outlining mismatches between the principles of the approach and psychological theory, advancements can be achieved.

In this paper a set of proposed modifications to the descriptive model are introduced, which allow inclusivity features such as distractions and limited cognitive ability to become part of the cognitive model. These new actions in the definition file may consequently be interpreted by the cognitive architecture, allowing for improvements in the accuracy of this method.

\section{Cognitive Architectures}

Cognitive architectures are toolkits that combine theories of cognition to simulate human behaviour. The simulation is not purely a model of the behavioural output of the human mind, but rather aim to replicate structural properties of the modeled system. These do not necessarily physically replicate components of the system - rather, virtual machines replicate the behaviour and knowledge of humans.

Originally, more advanced modeling tools (that included capabilities for simulated knowledge acquisition) required extensive experience with the modeling 
language and the underlying assumptions in order to accurately simulate cognitive processes. Recently, cognitive models have become more accessible to users that have limited or no modeling expertise 6]; examples for this include Salvucci and Lee's ACT-Simple [20] which uses a KLM-GOMS 12] based descriptive language to automatically construct ACT-R models. This approach is further emphasised by St. Amant and Ritter [21] who create ACT-R models based on the specification of the user interface and keyboard layout of mobile phones.

\section{$3 \quad$ Implicit Modeling}

In the process of translating an interface definition to a format that is interpretable by a cognitive architecture, the visual and interactive features of the interface (and the device on which the interface is displayed) must be simplified and expressed in a generic format. In this process (which, for the purposes of analysis may be equated with sampling) information about the interface is lost. The degree to which information is lost depends on whether the specifics of the interface can be described by the definition language used and if the variables, in which aspects of the interface are recorded, match the requirements set by an approximation of human behaviour through a cognitive simulation.

The above described translation procedure is summarised as an implicit modeling procedure because the process of translation includes disregarding elements of the user interface. Hence, a reduced amount of information about the interface design is passed on to the cognitive architecture, limiting the potential of the cognitive tool itself. Furthermore, by selecting specific criteria of the interface (e.g. the parameters of GOMS), the translation procedure has a significant effect on the modeling procedure.

\subsection{Limitations and Repurposing}

With the aim of implementing a variant of GOMS (Goals, Operators, Methods and Selection Rules) as the underlying description engine for a cognitive framework, the difference between traditional (CMN-) GOMS 3] and KLM [4] are of particular interest. CMN-GOMS (Card, Moran and Newell) consists of pseudocode with no formal syntax, is therefore very flexible and allows the definition of goals and subgoals. Methods within CMN are informal programmes and selection rules are defined in a tree structure where different branches are used for different scenarios. KLM-GOMS, on the one hand, is a simplified version of GOMS, where only keystroke-level operators are of interest to the framework and consequently place implicit focus on low-level actions by the user. There are no goals, methods or selection rules included in the model, therefore the definition is strictly sequential, making a KLM definition quick and easy but limited in scope.

Even though GOMS is a long-established route to defining interface and interaction routes, there are clear limitations when defining tasks. GOMS is only implementable with well-defined routine cognitive tasks and does not take error, 


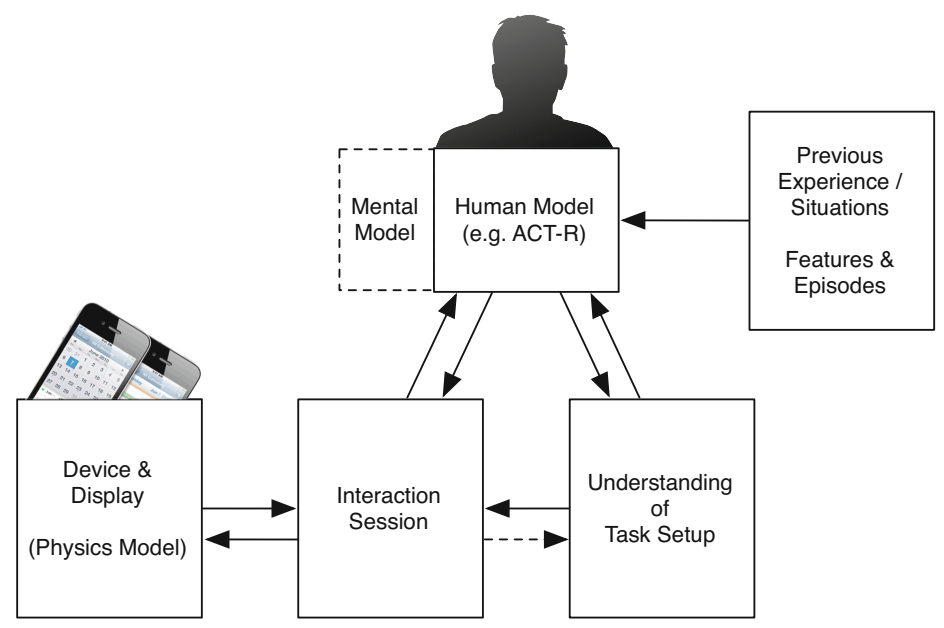

Fig. 1. An overview of what implicit modeling aims to achieve within the context of a simulated interaction between a human model and an interface definition

fatigue or differences in social surroundings into consideration, which are of particular interest when approaching ICT design processes from an inclusive design perspective.

John states that in the context of GOMS, operators were "command[s] and [their] parameters" [1] and at the time of her review (1995), "with graphic user interfaces, operators are just as likely to be menu selections, button presses, or direct manipulation actions" [11. In the future, John defines operators to include "gestures, spoken commands, or even eye movements" [11. With the growing prevalence of touchscreen interaction paradigms on mobile and desktop devices, John's prediction holds true, yet GOMS techniques have not adjusted to include the requirements set by touch- and gesture-based input.

There are three potential outputs from the described GOMS techniques: timing of the execution of individual tasks, the time required to learn a specific task and the likelihood of forgetting elements of the required knowledge for completing a task.

One of the greatest limitations of all GOMS implementations (in their current versions) is that they are aimed at users that have mastered the elements of the task that he/she is required to complete. It is assumed that the "cognitive skill" required for the completion of the goals in a procedure outlined in GOMS will be mastered by the user. This applies to individual operators in the context of GOMS, but does not predefine methods or goals laid out by a specific GOMS procedure. This limitation highlights the need for a more inclusive approach to implementing GOMS techniques in the design process of interfaces and touchscreen interfaces in particular.

John addresses this issue in her review of GOMS techniques as a whole; while she concludes that "[if] you are designing a system that will only have novice 
users, like a kiosk at the Olympics, then predictions of skilled behaviour are not necessary[; instead, it is] better to run a few representative users through a laboratory usability test or use Cognitive Walkthrough" [11. As discouraging as this statement may sound in the context of inclusive design research, John suggests to "look carefully: coming up with the action sequences that form the basis for a Cognitive Walkthrough is almost the same as doing a Keystroke-Level Model" [1].

\subsection{Need for Implicit Modeling}

The motivation for this project is based on a range of issues that have not been adequately addressed in industrial or scientific contexts. This includes the problems associated with setup procedures on (mobile) digital interfaces, their implications for widespread device use, cognitive modeling tools acting as a stagegate in the design process of inclusive user interfaces, the associated generational debate and last, but not least, the need for simplified usability evaluation techniques.

Stage-Gate in Use. The initial tasks on a mobile OS are setup procedures or require setup procedures to function. A user's perception of being able to use software is highly dependent on the success of his/her first interaction with the software. Literature suggests that the perception of the user to be able to use software is a key motivational factor [5] for further use. Given the importance of first-time use as a key element in motivating the digitally excluded in using new technology, this project focuses on establishing new design practices for touchscreen initial setup tasks and, more specifically, virtual validation tools in this field.

Setup tasks are a stage-gate in further device usage. Initial studies by Wollner et al. 23. with a range of users in different age groups further support this hypothesis. Hence, it is of importance to improve the design process of setup procedures as a whole (e.g. for all age groups and levels of ability), either by empowering designers with better testing tools, which can be employed throughout all stages of development and/or to replace testing procedures employed prior to product deployment. Prior experience plays a significant role in bypassing this stage-gate [14, but there is further research that could be supported through improved cognitive modeling frameworks. Finally, these improvements would allow for further analysis of the effects of the context of use [18] on usability.

Stage-Gate in the Design Process. The complexity of using cognitive modeling tools excludes many interface designers from more frequent use [19. By enhancing the sampling stage of user interface cognitive modeling, the overall process may be improved and hence made more accessible for interface design and development purposes. Concurrently, virtual user performance modeling tools in this field are constrained by the sampling procedure between the interface definition and the cognitive architectures they are evaluated by. This highlights the need for new interface sampling methods. 
Simplified Usability Evaluation. Based on the increasing need for solutions for independent living with digital interfaces, there is a clear need for streamlining usability evaluation practices in industrial applications [15]. Resources for the time- and resource-intensive process of testing (in-lab and in-field) of new ICT will, in the long run, decrease. A potential solution for this is employing digital models of a wide range of users, allowing (iterative) product development/design and the subsequent validation processes to be completed more quickly and efficiently.

\section{Proposed Changes}

The literature presented in the previous sections, changes in the interaction paradigms of modern interfaces and an increasing need for the adaption of inclusive design methodologies in the area of interface design, highlight the need for an adaption of implicit modeling to better sample interface designs.

The basis for the recommended changes outlined in this section is based on the pilot experimental work conducted with novice users attempting to complete a setup procedure on a range of mobile touchscreen implementations [23], shortcomings of current implementations in relation to the changing demands of mobile interfaces the need for implicit modeling that is scientifically validated and practically deployable to an increasingly diverse population of interface designers, and the lack of inclusive measures in current implicit modeling implementations.

An outline of cognitive difficulties with, and the limited access to information systems, as given by [8], may provide a basis of input to the proposed adaption of implicit modeling. Likewise, with an emphasis on mobile devices, the conceptual framework and models for identifying and organising usability impact factors, as outlined by [9], may provide further insights in the definition of an extension to implicit modeling techniques.

In the process of defining a GOMS model for mobile touchscreen devices that includes scope for slips, error, fatigue or differences in social parameters which we define as inclusive factors, there is a host of variables that need to be defined. Equally, the constraints of new (touchscreen) interaction paradigms and a larger variance in screen sizes allows the definition of new operators. We choose KLM-GOMS as a definition basis because user modeling and validation requires a stringent definition, which has already been implemented in tools such as CogTool. Further development aims at identifying which parameters are missing from a range of perspectives, as outlined in the following sections.

\subsection{Adaption for Inclusivity}

The fundamental purpose of this project is to extend the inclusivity of cognitive modeling approaches in order to better approximate the needs and limitations of a wider demography compared to what is possible with current modeling approaches. This includes several explicit measures that directly relate to the 
diversity of potential users of the technology that is being assessed with the simulation tool as well as implicit measures, which, overall, are able to improve the accuracy of modeling techniques - given the current state of technology.

One of the most significant challenges in adapting implicit modeling to include a wider range of capabilities and hence make implicit modeling explicitly more accommodating for a more diverse population of users, is to include parameter estimation for the interfaced cognitive architecture. This can be accomplished by providing a range of interface definitions in the implicit modeling approach, each with a weighting that relates to age- and capability-related limitations of the user. A further method would be adjusting the parameters that dictate cognitive ability, memory retention and factors of persistence (intrinsic motivation) in the cognitive architecture directly.

This also raises the question whether the framework's output should aim to establish a population mean for performance or whether it should integrate a range of capability levels in order to provide a more in-depth analysis of the inclusivity of a specific interface design. The next stages of our research will focus on the topic of parameter estimation - both in the implicit model as well as by passing parameters on to the interfaced cognitive architecture - and aim to develop a robust methodology that provides an insight into inclusivity factors without compromising the approach's feasibility in common design practices. A further aim of the adaption is the approximation of exclusion statistics for individual components of a simulated interface interaction.

\subsection{Adaption for Touchscreen Interfaces}

Another substantial limitation of today's implementation of implicit modeling is the lack of adaption to touchscreen interfaces. This is of great significance due to the high volume and quick turnover of the development of touchscreen interfaces, which are used by an ever increasing and diversifying population of users. Especially in the development of touchscreen interfaces - both on dedicated devices as well as in embedded systems - the design process is in need of a robust modeling-based evaluation tool that can assess potential shortcomings of designs at an early developmental state.

In its current state, KLM-GOMS does not support the definition of gesturebased input - a factor, especially from the perspective of its potential lack of inclusivity, that has become increasingly important. Touchscreens are also unique in terms of the wide array of available screen sizes and responsiveness. These are all factors that are currently not definable by a KLM-GOMS description of the interface.

Combining the two above-mentioned properties leads to the zooming and scrolling functions that are unique to touchscreen interfaces and are currently not describable by KLM-GOMS interfaces. For instance, the double-tap to zoom implementation on most mobile touchscreen systems is of vital importance from an inclusivity perspective (both from a functional and conceptional standpoint) but fails to be easily describable in current GOMS literature. 
Another significant factor affecting the user's performance is the absence of modeling finger- and hand-based occlusions when operating the device. Here, various touchscreen implementations have offset controls (mimicking an indirect interaction paradigm) when, for instance, selecting a letter on the keyboard. The issues of hand- and finger-based occlusions are, again, not describable using a KLM-based approach.

A further usability-centred variable is the presence of on-screen distractions and the method in which they are describable by a KLM-GOMS definition. Despite the fact that distractions have been present since the existence of graphical user interfaces, the number of off-task distractions is especially large on current (touchscreen) interfaces due to a stark increase in interface-based user interrupts (e.g. a pop up or notification abut a message when looking at photos). Likewise, the prevalence of system-wide notification systems, as implemented by most mobile operating systems is an on-screen distraction which, equally, cannot be modeled by the status quo of KLM-GOMS. Here, we present two types of distractions: on- and off-task. On-task distractions relate to graphical features, which disrupt the users workflow but directly relate to the task, such as oversized additional options or colour-based distraction in a specific UI-flow. Offtask distractions relate to on-screen distractions which distract the user from the task and do not relate to the task, such as system-wide notifications or pop-ups relating to another process on the device.

Finally, a further, touchscreen-specific element that dictates a new definition in KLM-GOMS are finger-based requirements on what is described as "homing" in the original definition of KLM-GOMS. Whilst defined in previous implicit modeling implementations, it was previously based on the accelerated indirect interaction paradigm of mouse control.

\subsection{Adaption for Mobile Environments}

Previous work that may be described as implicit modeling was based on dedicated workstations with large screens. In this environment, there was little variability of the effect of external factors on operating the device. With a growing presence of user interfaces that are used in mobile environments, the conditions of the environment have not only become more variable but also more distracting to the task the user aims to accomplish. Hence, there is a need for implicit modeling to be able to describe both the environment and the degree of distractions of the modeled interface.

This adaption of implicit modeling not only improves the accuracy of simulating the use of the described user interfaces in mobile environments but also allows for modeling in specific environments. This is of particular interest when adapting touchscreen interfaces for elderly users, which oftentimes struggle using the devices in mobile environments.

Adaptions for the mobile domain exist by Dunlop and Crossan [7, who tried applying the standard KLM operators to different types of text entry systems of mobile phones that were later modified by How and Kan [10] with adaptions that lead to better results through 13 new operators, which map to the key 
layout of a standard feature phone. Further work by Pavlovych and Stürzlinger 17. subsequently examined a similar issue (text entry on mobile phones) of nonexpert users based on the cognitive workload analysed by GOMS. The work completed compares different types of KLM-GOMS implementations for mobile environments. In our implementation, a combination of the above introduced literature builds a basis for adapting implicit modeling to the conditions set by mobile environments.

\section{Conclusion}

In this paper we propose a novel approach to modeling user behaviour in the context of touchscreen interface design. Building upon existing and constantly evolving cognitive architectures, which unify cognitive theories and allow the simulation of human behaviour, we propose a method of analysing the usability of touchscreen interfaces.

In order to achieve this, we define implicit modeling to be the translation procedure from an interface definition to the input of a cognitive architecture. Whilst elements of this translation procedure exist - such as KLM-GOMS - the current approach is inadequate. Part of the proposal is to extend KLM-GOMS in order to account for the requirements set by touchscreen interfaces, inclusivity and mobile environments and, in a further step, to integrate these changes into a toolkit that will support designers.

Further work includes implementing a prototype translation tool, testing this prototype and validating the resultant data with real users. Likewise, once implemented, exploring the ways in which the simulation output of the cognitive architecture may be used for better analysing the UI defines an important next step. One such approach may be by employing Graph Theory, as previously explored by Thimbleby [22].

\section{References}

1. Anderson, J.R., Bothell, D., Byrne, M.D., Douglass, S., Lebiere, C., Qin, Y.: An integrated theory of the mind. Psychological Review 111, 1036-1060 (2004)

2. British Standards Institution: Managing Inclusive Design. Number BS 7000-6:2005. In: Design management systems, London (2005)

3. Card, S.K., Moran, T.P., Newell, A.: The Psychology of Human-Computer Interaction. Erlbaum (1983)

4. Card, S.K., Moran, T.P., Newell, A.: The Keystroke-Level Model for User Performance Time with Interactive Systems. Commun. ACM 23(7), 396-410 (1980)

5. Compeau, D.R., Higgins, C.A.: Application of Social Cognitive Theory to Training for Computer Skills. Information Systems Research 6(2), 118-143 (1995)

6. Councill, I.G., Haynes, S.R., Ritter, F.E.: Explaining Soar: Analysis of Existing Tools and User Information Requirements. In: Proceedings of the Fifth International Conference on Cognitive Modeling (2003)

7. Dunlop, M.D., Crossan, A.: Predicitve Text Entry Methods for Mobile Phones. Personal Technologies 4(2-3) (2000) 
8. Gregor, P., Dickinson, A.: Cognitive difficulties and access to information systems: an interaction design perspective. Universal Access in the Information Society 5(4), 393-400 (2006)

9. Ham, D., Heo, J., Fossick, P., Wong, W., Park, S., Song, C., Bradley, M.: Conceptual framework and models for identifying and organizing usability impact factors of mobile phones. In: Proceedings of the 20th Conference of the Computer-Human Interaction Special Interest Group (CHISIG) of Australia on Computer-Human Interaction Design: Activities, Artefacts and Environments - OZCHI 2006, Sydney, Australia, p. 261 (2006)

10. How, Y., Kan, M.Y.: Optimizing Predicitive Text Entry for Short Message Service on Mobile Phones. In: HCII 2005 (2005)

11. John, B.E.: Why GOMS? Interactions 2(4), 80-89 (1995)

12. John, B.E., Prevas, K., Salvucci, D.D., Koedinger, K.: Predictive human performance modeling made easy. In: Proceedings of the SIGCHI Conference on Human Factors in Computing Systems, CHI 2004, pp. 455-462. ACM, New York (2004)

13. Kieras, D.E.: Using the Keystroke-Level Model to estimate execution times. University of Michigan (2001)

14. Langdon, P., Lewis, T., Clarkson, P.J.: The effects of prior experience on the use of consumer products. Universal Access in the Information Society 6(2), 179-191 (2007)

15. Office for National Statistics: Statistical bulletin: Internet Access - Households and Individuals (2012), http://www.ons.gov.uk/ons/rel/rdit2/

internet-access--households-and-individuals/2012/ stb-internet-access-households-and-individuals-2012.html

16. Osterloh, J.P., Feil, R., Ludtke, A., Gonzalez-Calleros, J.: Automated UI Evaluation based on a Cognitive Architecture and UsiXML, pp. 1-9 (2011)

17. Pavlovych, A., Stürzlinger, W.: Model for Non-expert Text Entry Speed on 12button Phone Keypads. In: CHI 2004, pp. 351-358. ACM Press (2004)

18. Persad, U., Langdon, P., Clarkson, P.J.: Characterising user capabilities to support inclusive design evaluation. Universal Access in the Information Society 6(2), 119-135 (2007)

19. Ritter, F.E.: Some Frontiers of Cognitive Modeling: A Modest Research Agenda Exploring Emotions and Usability (2008)

20. Salvucci, D.D., Lee, F.J.: Simple Cognitive Modeling in a Complex Cognitive Architecture. In: Proceedings of CHI 2003, Human Factors in Computing Systems, pp. 265-272. ACM Press (2003)

21. St Amant, R., Ritter, F.E.: Automated GOMS-to-ACT-R Model Generation. In: International Conference on Cognitive Modeling, Lea, Mahwah, NJ, pp. 28-34 (2004)

22. Thimbleby, H., Gow, J.: Applying Graph Theory to Interaction Design. In: Gulliksen, J., Harning, M.B., Palanque, P., van der Veer, G.C., Wesson, J. (eds.) EIS 2007. LNCS, vol. 4940, pp. 501-519. Springer, Heidelberg (2008)

23. Wollner, P.K.A., Goldhaber, T.S., Mieczakowski, A., Langdon, P., Clarkson, P.J.: Evaluation of setup procedures on mobile devices based on users' prior experience. In: NordDesign 2012 (2012) 